\title{
Vehicular Traffic Surveillance and Road Lane Detection Using Radar Interferometry
}

\author{
David Felguera-Martín, José-Tomás González-Partida, Pablo Almorox-González, and Mateo Burgos-García
}

\begin{abstract}
Speed enforcement on public roadways is an important issue in order to guarantee road security and to reduce the number and seriousness of traffic accidents. Traditionally, this task has been partially solved using radar and/or laser technologies and, more recently, using video-camera based systems. All these systems have significant shortcomings that have yet to be overcome. The main drawback of classical Doppler radar technology is that the velocity measurement fails when several vehicles are in the radars beam. Modern radar systems are able to measure speed and range between vehicle and radar. However, this is not enough to discriminate the lane where the vehicle is driving on. The limitation of several vehicles in the beam is overcome using laser technology. However, laser systems have another important limitation: They cannot measure the speed of several vehicles simultaneously. Novel video-camera systems, based on license plate identification, solve the previous drawbacks, but they have the problem that they can only measure average speed but never top-speed. This paper studies the feasibility of using an interferometric linear frequency modulated continuous wave radar to improve top-speed enforcement on roadways. Two different systems based on down-the-road and across-the-road radar configurations are presented. The main advantage of the proposed solutions is they can simultaneously measure speed, range, and lane of several vehicles, allowing the univocal identification of the offenders. A detailed analysis about the operation and accuracy of these solutions is reported. In addition, the feasibility of the proposed techniques has been demonstrated with simulations and real experiments using a Ka-band interferometric radar developed by our research group.
\end{abstract}

Index Terms-Automotive radar, road traffic control, road vehicle location monitoring, road vehicle radar, traffic control (transportation).

\section{INTRODUCTION}

$\mathbf{N}$ OWADAYS, traffic surveillance is an important civilian application to improve road control, law enforcement, intelligent roads, and urban congestion. Several works analyze this problem and propose different solutions to deal with this task [1]-[4]. In the framework of traffic surveillance, an outstanding issue is the speed control on roadways. There are several technologies to carry out speed measurements of noncooperative vehicles.

Doppler radar in down-the-road (DTR) configuration has been widely used for speed enforcement on public roadways [5], [6]. In DTR configuration, the beam of the antenna is directed along the line of travel of the target vehicle. The main shortcoming of DTR Doppler radar is target identification [7]. Doppler radar devices are not target selective since they have a wide beamwidth, and the speed measurement is erroneous when there are two or more targets in the radar beam [8].

An alternative to minimize this drawback is the use of Doppler radar in across-the-road (ATR) configuration [7], [9]. This scheme is employed to measure vehicle speeds by directing the microwave beam across, instead of down (or along), the road. The main advantage over DTR configuration is that the operational area of its beam is reduced. This reduces the target-identification problem and also decreases the probability of more than one vehicle will be in the beam at the same time. However, these assumptions fail when traffic volume is very dense. Furthermore, there is an added complexity to ATR radar: the cosine effect. Doppler radar measures the radial velocity of the target vehicle, i.e., what is measured is the speed of the target vehicle multiplied by the cosine of the angle between the beam and the direction of motion. The radar device can correct this cosine effect but only if the angle is known. Thus, the cosine effect introduces a new uncertainty into the measurement process and, in most cases, a calibration is needed [9].

Laser traffic radars in DTR configuration solve the problem of target identification [10], [11]. These devices have a very narrow beam width, thereby enabling the selection of individual vehicles. These systems measure the speed using the delay history of a burst of laser pulses. The main drawback is that the system has to concentrate on a unique target at a time, i.e., a different laser is needed for every road lane in order to control the complete roadway. In addition, an assumption is made for a right performance: the laser pulses would all strike a flat surface perpendicular to the path of the light wave. However, in practice, the waves strike the front or rear of the vehicle which are irregular surfaces. The result is a reflected laser pulse that is dispersed in time due to the different transit times for different portions of the reflected beam. It is also dispersed in angle. Hence, multiple reflections from neighboring objects can further confuse the measurement [7].

Unlike DTR laser devices, ATR laser systems employ two laser beams and operate on the time-distance principle [7]. The lasers are mounted on a horizontal bar and transmit parallel light beams that are separated by a known distance. The equipment directs the beams across the roadway perpendicular 
to the direction of traffic flow. A beam detects a vehicle by sensing changes in the intensity of reflected light. Since the distance between the beams is known, the transit time for the vehicle front edge from one beam to the next one is easily converted into a speed measurement by dividing it into that known distance. The major limitation of this system is that it only measures the speed of one target at a certain time, and if two vehicles in adjacent lanes overlap each other, the system fails.

Other works have proposed range-Doppler radars to solve the problem of several vehicles in the radar beam [12], [13]. These systems can discriminate the echoes of vehicles located at different distances to the radar. In that sense, the system improves the capabilities of current Doppler or laser traffic radars, which have to concentrate on a unique target at a time. However, the association of each echo, or speed measurement, to each target has yet to be solved. This is because two targets located at different road lanes could be detected at the same range bin by the radar.

Currently, camera-based systems are used to measure the mean speed in a stretch. One camera registers the license plate of each vehicle that drives in the stretch, saving the transit time. The other camera registers the license plate of the same vehicle that drives out the stretch, saving the transit time, too. Knowing the distance between both cameras and both transit times, the system computes the average velocity of each vehicle. The main shortcoming of this system is that only computes the average speed, but it cannot measure the top speed of the vehicles. A second drawback is that these systems can be only installed in road stretches without entrances or/and exits.

Another alternative proposed in the literature for collision warning, collision avoidance, and adaptive or intelligent cruise control is a monopulse Doppler radar [14]. This is a Doppler radar system that uses a monopulse antenna scheme to track multiple targets and generate range, velocity, and azimuth angle output data for each vehicle. This system uses two different frequencies to measure range, and a monopulse antenna to measure the azimuth angle. This system could be easily adapted to speed control applications. The association of each echo to each target could be partially solved with that system, i.e., two targets located at different road lanes could be discriminated by the azimuth and range measurements. However, this system can only discriminate targets with different speeds, and therefore, if two vehicles are located at different road lanes and/or distances, but they travel with the same velocity, the system fails. Furthermore, the range measurement suffers ambiguity because it is computed from a phase difference.

Here, we propose two different schemes that solve the shortcomings of all previous systems. These schemes solve, with a unique radar, the association of each echo to each target. Both schemes are based on an interferometric linear frequency modulated continuous wave (LFMCW) radar. The proposed schemes can measure range, speed, and azimuth of several targets in the beam at the same time, differentiating and identifying all of them. Therefore, these schemes solve the classical shortcoming of target identification, allowing the simultaneous top-speed measurement of several vehicles with a unique system.
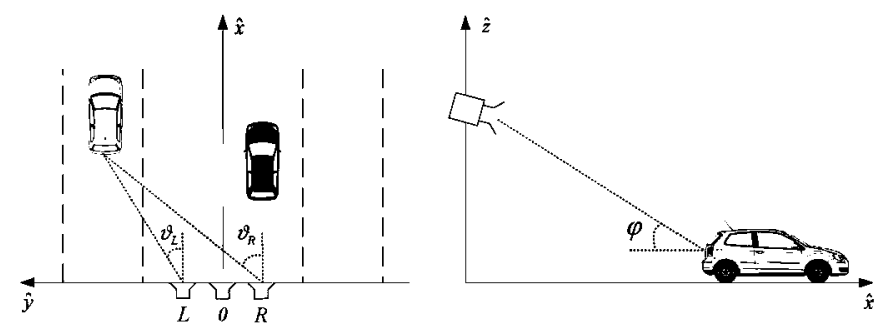

Fig. 1. Down-the-road detection geometry.

This article explains carefully the operation of the interferometric LFMCW radar in two configurations: DTR and ATR. In addition, a sensitivity analysis of the measurements accuracy is presented for both schemes. Both configurations have been experimentally characterized using simulated and real radar data from an interferometric LFMCW demonstrator [15] operating at Ka band ( $35 \mathrm{GHz})$.

\section{Down-The-Road Configuration}

\section{A. Geometry}

In DTR configuration, the axis of transmitting and receiving antennas is directed along the line of travel of the target vehicle. This direction will be the same as the road direction and is represented with the $\hat{x}$ axis in Fig. 1. In our detection geometry, the transmitting antenna is located at $O$, and the receiving antennas are at $L$ and $R$. Viewed from the receiving positions the different targets will have azimuth angles represented by $\theta_{L}$ and $\theta_{R}$ and elevation angles denoted by $\varphi_{L}$ and $\varphi_{R}$.

\section{B. Road Lane and Speed Detection}

In a conventional DTR radar, the target radial speed $V_{r}$ is measured using the Doppler shift $f_{d}$ caused in the received signal using (1), where $\lambda$ is the radar central wavelength. However, there is no way to identify which vehicle is speeding in case two of them are inside the beam. Using high-resolution radars, we can estimate motion parameters for all the illuminated targets, but we cannot determine which echo corresponds to each vehicle [13]. Another problem with DTR high-resolution radars is that the target response is spread among multiple Doppler frequencies introducing a high uncertainty in speed measurement which increases with increasing speed [13]

$$
V_{r}=-\frac{\lambda}{2} f_{d}
$$

In order to overcome the issue related with Doppler spread, we use the target range history $r(t)$ to estimate the radial velocity (2), as proposed in [13]. We use the range profile alignment algorithm described in [16] to obtain the range history. This algorithm is robust against changes in the strongest return of the target, and it allows to measure accurately the velocity of the target during the dwell time

$$
V_{r}=\left\langle\frac{\partial r(t)}{\partial t}\right\rangle \text {. }
$$

In order to identify which radar echo corresponds to each target vehicle, we use the difference in the measured radial 
velocity from the receiving antennas, which are located in slightly different positions. The difference in measured radial velocity between antennas depends on the road lane where the target is located. This fact can be used to identify the vehicle lane. However, this difference will be very small, usually less than the Doppler resolution cell, and in order to measure it accurately, the phase difference of the received signals needs to be used. The absolute phase difference between the antennas, or interferometric phase, is related to the target distance to each antenna, $r_{L}$ for the left and $r_{R}$ for the right one, and is given by

$$
\phi_{I}(t)=\frac{2 \pi}{\lambda}\left(r_{L}(t)-r_{R}(t)\right) .
$$

Calculating the gradient of the interferometric phase, it can be seen that it is related with the difference in radial velocities between the antennas, $v_{r_{L}}$ for the left and $v_{r_{R}}$ for the right one, using

$$
\begin{aligned}
\frac{\partial \phi_{I}(t)}{\partial t} & =\frac{2 \pi}{\lambda}\left(\frac{\partial r_{L}(t)}{\partial t}-\frac{\partial r_{R}(t)}{\partial t}\right) \\
& =\frac{2 \pi}{\lambda}\left(v_{r_{L}}(t)-v_{r_{R}}(t)\right) .
\end{aligned}
$$

If a target with constant velocity and no acceleration is considered, which is typical for the small dwell time of DTR radars, equation (5) is obtained, that relates radial velocity difference to along the road speed $V_{x}$ and azimuth and elevation angles

$v_{r_{L}}(t)-v_{r_{R}}(t)=V_{x} \cos \theta_{L}(t) \cos \varphi_{L}(t)-V_{x} \cos \theta_{R}(t) \cos \varphi_{R}(t)$.

If we assume that the distance to the target will be much greater than the height of the radar location, $x \gg h$, we can relate the difference in azimuth angles to the interferometric phase gradient in

$$
\frac{\partial \phi_{I}(t)}{\partial t} \simeq \frac{2 \pi}{\lambda} V_{x}\left(\cos \theta_{L}(t)-\cos \theta_{R}(t)\right) .
$$

The absolute interferometric phase, $\phi_{I}$, cannot be measured by the radar, because it only measures the wrapped interferometric phase, $\psi_{I}$. Absolute and wrapped phases are related by (7). The absolute phase difference can be obtained from the wrapped one using phase unwrapping and calibration with a phase reference point

$$
\phi_{I}(t)=\psi_{I}(t)+2 \pi n(t)+\phi_{0} \quad(n \in N) .
$$

However, an absolute estimation of the interferometric phase is not necessary for our system as long as the interferometric phase gradient is used. The absolute and measured phase gradient are equal for all phase values except when there is a $-\pi$-to- $\pi$ phase shift, where a change of $n(t)$ between samples occurs. In that samples, an outlier will be present, but it can be easily removed. Problems related to phase unwrapping and phase calibration are avoided with this technique

$$
\frac{\partial \phi_{I}(t)}{\partial t}=\frac{\partial\left(\psi_{I}(t)+2 \pi n(t)+\phi_{O}\right)}{\partial t} \simeq \frac{\partial \psi_{I}(t)}{\partial t} .
$$

Using (6) and prior knowledge of the angles of lane limits as viewed from both receiving antennas, we can establish a detection scheme to determine in which lane the target vehicle is driving

$$
\cos \theta_{L}(t)-\cos \theta_{R}(t) \simeq \frac{\lambda}{2 \pi V_{x}} \frac{\partial \phi_{I}(t)}{\partial t} .
$$

However, it is more practical to use a detection scheme based on the estimation of target cross-road position, $y$. Considering $x \gg y$ and $x \gg B$, being $B$ the baseline between the two receiving antennas, which is true in a typical detection scenario, the cosines of azimuth angles can be approximated by

$$
\begin{aligned}
\cos \theta_{L} & =\frac{x}{\sqrt{x^{2}+(y+B / 2)^{2}}}=\frac{1}{\sqrt{1+\left(\frac{y+B / 2}{x}\right)^{2}}} \\
& \simeq 1-\frac{1}{2}\left(\frac{y+B / 2}{x}\right)^{2} \\
\cos \theta_{R} & =\frac{x}{\sqrt{x^{2}+(y-B / 2)^{2}}}=\frac{1}{\sqrt{1+\left(\frac{y-B / 2}{x}\right)^{2}}} \\
& \simeq 1-\frac{1}{2}\left(\frac{y-B / 2}{x}\right)^{2} .
\end{aligned}
$$

Using (6), (10) and (11), the target cross-road position can be related to the measured interferometric phase gradient and, finally, isolate the $y$ coordinate of target vehicle obtaining

$$
y \simeq-\frac{\lambda x^{2}}{2 \pi V_{x} B} \frac{\partial \phi_{I}}{\partial t} .
$$

An initial estimation of the road lane can be done assuming $x \simeq R$ and $V_{x} \simeq V_{r}$, where $R$ and $V_{r}$ are the measured distance and radial velocity of the target in any of the receiving antennas. Once we know the initial estimated lane, a better estimation of $y$ and $V_{x}$ can be done by correcting the cosine effect using prior knowledge of the azimuth and elevation angle for the detected distance and lane

$$
V_{x}=\frac{V_{r}}{\cos \theta \cos \varphi} .
$$

\section{Uncertainty in Road Lane Measurement}

1) Quantitative Analysis: The uncertainty of the cross-road position estimation will be affected by the uncertainty of several other magnitudes. These magnitudes are phase difference, speed estimation, $x$ position, and central wavelength. Assuming the uncertainty sources are linearly independent, the total uncertainty is given by

$$
\sigma_{y}^{2}=\left|\frac{\partial y}{\partial \dot{\phi}_{I}}\right|^{2} \sigma_{\dot{\phi}_{I}}^{2}+\left|\frac{\partial y}{\partial V_{x}}\right|^{2} \sigma_{V_{x}}^{2}+\left|\frac{\partial y}{\partial x}\right|^{2} \sigma_{x}^{2}+\left|\frac{\partial y}{\partial \lambda}\right|^{2} \sigma_{\lambda}^{2} .
$$

Using (12) and operating, we finally obtain

$$
\sigma_{y}^{2}=\left|\frac{\lambda x^{2}}{2 \pi V_{x} B}\right|^{2} \sigma_{\dot{\phi}_{I}}^{2}+\left|\frac{y}{V_{x}}\right|^{2} \sigma_{V_{x}}^{2}+\left|\frac{2 y}{x}\right|^{2} \sigma_{x}^{2}+\left|\frac{y}{\lambda}\right|^{2} \sigma_{\lambda}^{2} .
$$


TABLE I

DTR Simul,ateid SClinario Charactiristicsand Risuits

\begin{tabular}{ccccccccc}
\hline $\begin{array}{c}\text { Target } \\
\text { number }\end{array}$ & $\begin{array}{c}\text { Initial } \\
\text { along-road } \\
\text { position }(\mathrm{m})\end{array}$ & $\begin{array}{c}\text { Actual } \\
\text { Speed } \\
(\mathrm{Km} / \mathrm{h})\end{array}$ & $\begin{array}{c}\text { Actual } \\
\text { cross-road } \\
\text { position }(\mathrm{m})\end{array}$ & $\begin{array}{c}\text { Actual } \\
\text { Lane }\end{array}$ & $\begin{array}{c}\text { Detected } \\
\text { Range } \\
(\mathrm{m})\end{array}$ & $\begin{array}{c}\text { Detected } \\
\text { Speed } \\
(\mathrm{Km} / \mathrm{h})\end{array}$ & $\begin{array}{c}\text { Detected } \\
\text { cross-road } \\
\text { position }(\mathrm{m})\end{array}$ & $\begin{array}{c}\text { Detected } \\
\text { Lane }\end{array}$ \\
\hline 1 & 25.0 & 115.0 & -5.25 & 4 & 28.90 & 114.91 & -5.26 & 4 \\
2 & 35.0 & 170.0 & -5.25 & 4 & 40.10 & 170.20 & -5.10 & 4 \\
3 & 44.0 & 105.0 & -1.75 & 3 & 48.50 & 105.00 & -1.89 & 3 \\
4 & 55.0 & 120.0 & -1.75 & 3 & 59.20 & 120.02 & -1.63 & 3 \\
5 & 45.0 & 90.0 & +1.75 & 2 & 48.20 & 90.03 & +1.78 & 2 \\
6 & 55.0 & 80.0 & +5.25 & 1 & 57.90 & 80.06 & +5.26 & 1 \\
\hline \hline
\end{tabular}

In addition to these uncertainties, there is another one related to the calibration technique used to periodically remove any constant errors [5].

2) Second-Order Effects: The uncertainty in cross-road position is computed using (15). We will focus our analysis in the term related to the uncertainty in the phase difference measurement, which is the only term that is unique to interferometric radars. Due to the approximations made in formulas derivation, some errors affecting sensitivity are still present. These errors cannot be evaluated in a quantitative way because their influence is not seen in the approximated mathematical expression (12). However, these errors can still be analyzed in a qualitative way.

The first assumption, i.e., $x \gg h$, is neglecting the elevation angle in the derivations. However, it is easy to see that decreasing the height of the radar will result in a decrease in the radial velocity difference and, as seen in (15), in a higher uncertainty in the cross-road position measurement. Other geometrical assumptions, i.e., $x \gg y$ and $x \gg B$, make impossible to evaluate the dependence in the cross-road position uncertainties with the cross-road position itself. However, it will be seen in Fig. 3(a) that the road lane limits in the detection map get slightly closer when the values of the cross-road position are increased, and hence, there is more uncertainty in the cross-road position. In previous formulas derivation, a target with constant velocity and no acceleration has been considered. When the zero acceleration assumption is not met, it will produce an slightly variation in radial velocity difference and, as a result, an increase in system uncertainties.

\section{Operational Limits}

In order to obtain the gradient of interferometric phase, that we need to estimate the cross-road position with (12), the phase change between consecutive samples must be smaller than $\pi$ radians. This condition will set the maximum bounds for target speed to correctly detect the lane. Using (12), and replacing the continuous phase derivative by time and phase increments, we obtain (16), shown below, where the time increment is the inverse of the ramp repetition frequency $(R R F)$

$$
\begin{aligned}
V_{x} & \simeq-\frac{\lambda x^{2}}{2 \pi B y} \frac{\Delta \phi_{I}}{\Delta t} \\
\Delta t & =\frac{1}{R R F} .
\end{aligned}
$$

The restriction to correctly estimate the phase gradient is having two samples in each phase cycle. However, in order to get the best performance of this technique, and reduce phase difference variance, is better to have more samples per phase cycle

$$
\Delta \phi_{i}=\frac{2 \pi}{N_{s}} .
$$

Finally, if we consider $N_{S}$ samples per phase cycle, the maximum speed to correctly estimate the road lane will be given by

$$
V_{x \max } \simeq \frac{\lambda x^{2}}{B y} \frac{R R F}{N_{S}} .
$$

An intrinsic limitation of DTR configuration is that two or more targets simultaneously traveling with equal speed and at the same range regarding the radar will be indistinguishable no matter the lane the vehicles are located. Some conclusions can be stated when looking at (19). It is possible to detect higher velocities in the central lanes than in the outer ones. A higher distance to the radar means a higher detectable velocity. The maximum detectable velocity will increase if the ramp repetition frequency or the wavelength is increased. It can be also increased if the baseline or the number of samples per phase cycle is decreased. However, all these parameters will have different effects in the uncertainties so a tradeoff must be reached.

\section{E. Simulation Results}

In this section, three kind of simulation results are presented. In first place, we will show the correct performance of the proposed interferometric DTR configuration in a dense traffic scenario. This simulation is also used to illustrate the signal processing chain. In second place, we will validate the analytic formula for the uncertainty in the cross-road position measurement with a Monte Carlo simulation. Finally, we will use another Monte Carlo simulation to show the performance against noise.

In a dense traffic scenario, there will be typically more than one target in the radar beam along the same lane or in different road lanes. We have placed six targets in a four lane highway in this simulation. The initial positions and speed of the targets can be seen in Table I along with the results of the detected range, speed, and road lane. The first stage of the signal processing chain is to obtain a range-time image of the scene from each of the receiving antennas. The images for our example are shown in Fig. 2. These images will allow us to estimate the targets velocity and obtain the phase difference in order to estimate the cross-road position and determine the road lane of the target. As we stated before, a range profile alignment algorithm is used to estimate the target speed. Then, the corresponding 


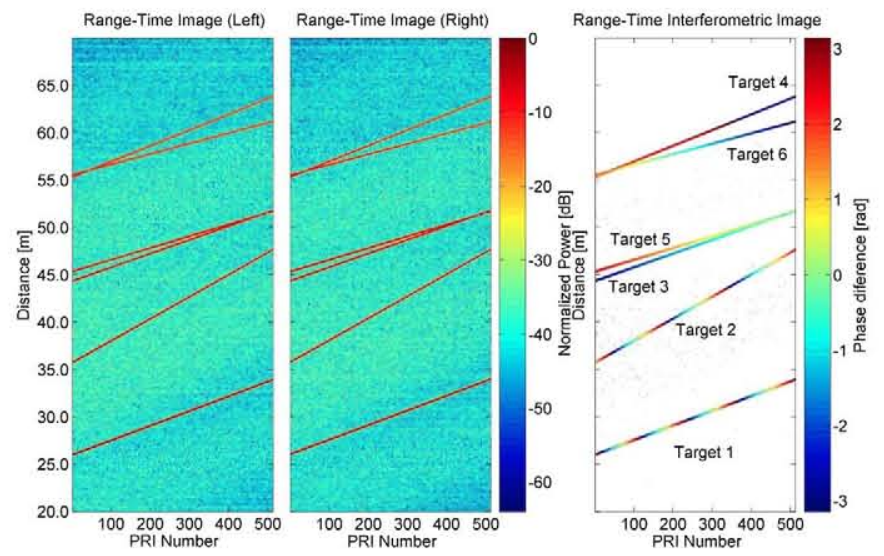

Fig. 2. DTR range-time images in a simulated scenario.

interferometric phase is extracted in an easy way once the target profiles are already aligned. Isolating the targets is necessary to correctly perform the profile alignment. Hence, prior to the alignment and phase extraction, the targets in the range-Doppler domain are isolated and then converted back to the range-time domain. Speed estimation is trivial, using (2) and (13), as soon as the evolution of the mean target position is obtained with the range profile alignment algorithm. Once the interferometric phase is extracted, the estimation of the cross-road position is also straight forward using (12).

A detail of the cross-road position estimation and the road lane detection map are shown in Fig. 3. The radial velocity difference detection map is shown in Fig. 3(a), and the position detection map is shown in Fig. 3(b). In these detection maps, the actual and the detected values for each target have been included. The limits of the road lanes are plotted in black, the actual target value is plotted in blue, and the detected target value is plotted with a different color for each target.

In order to plot all targets in the same figure, the radial velocity difference has to be normalized using the along-theroad target speed. Otherwise, a faster target will present a higher radial velocity difference, as it was stated in Section II-C. Fig. 3 clearly shows many of the reasonings made in Section II-C. Specifically, those related to the width of road lane limits in the radial velocity difference detection map. Furthermore, it can be clearly appreciated how a constant phase difference error in Fig. 3(a) is translated into a higher or lower uncertainty in cross-road measurement depending on target position in Fig. 3(b). In order to validate the analytic formula for the uncertainty in the cross-road position measurement, a Monte Carlo simulation has been run. In this simulation, we focus on the dependence of cross-road uncertainty with the distance to the radar, which is the predominant term of the factor associated to the phase difference gradient uncertainty. The simulation has been repeated for four different lanes. In Fig. 4, it can be seen how the analytic formulas agree with the obtained simulation results. The baseline used in the simulations is $B=0.77 \mathrm{~m}$. The central wavelength is $\lambda=8.6 e-3 \mathrm{~m}$, and the local oscillator has a typical phase error deviation of $\sigma_{\Phi}=0.1897 \mathrm{rad}$, which leads to a deviation in the interferometric phase difference of $\sigma_{\dot{\phi}_{I}}=0.3794 \mathrm{rad} / \mathrm{s}$. The measured standard deviation in speed measurement is $\sigma_{V_{x}}=8 e-3 \mathrm{~m} / \mathrm{s}$, and $\sigma_{x}=3 e^{-2} \mathrm{~m}$ in distance measurement. The deviation of the central wavelength is neglected in the simulations.

The performance of the DTR technique against noise is shown in Fig. 5. Two kind of curves are obtained using simulation, probability of detection, and classification against signalto-noise ratio (SNR). The SNR is defined in the time domain as the relation of the peak returned echo power and the noise power. We considered that a target is detected when we know about its presence and the error in the measured speed is less than a threshold. A target is classified when it is detected, the speed error is below the threshold, and the road lane is correctly estimated. In the DTR configuration, interferometry is used to estimate road lane position. It is interesting to point out that for a correct road lane classification, we need higher SNR than for just detecting it. In this example, we have used the same parameters as in previous simulations but for an isolated target with $120 \mathrm{Km} / \mathrm{h}$ speed. The maximum error allowed in the estimated speed is $3 \mathrm{Km} / \mathrm{h}$. For classification, in those conditions, we need an SNR $4 \mathrm{~dB}$ higher than for detection to correctly estimate the road lane.

\section{F. Experimental Results}

The experiments have been conducted with an interferometric LFMCW demonstrator [15] operating at $\mathrm{Ka}$ band $(35 \mathrm{GHz})$ in a controlled road scene. The transmitted bandwidth is $1600 \mathrm{MHz}$, and the ramp repetition frequency is $1000 \mathrm{~Hz}$. The baseline of our demonstrator is $77 \mathrm{~cm}$, and the receivers azimuth beam width is $6^{\circ}$. These are not the optimum values for this kind of configuration where longer baselines and azimuth beam widths are desirable for greater precision and coverage, respectively. However, our radar beam width still allowed us to test the system in a two-lane road shown in Fig. 6.

The field trials were conducted in a controlled two-lane road using two different types of vehicles. The first vehicle was a compact car (CC) and the other a sport utility vehicle (SUV). The two vehicles were approaching the radar in different lanes and with different speeds. The $\mathrm{CC}$ was located in the left lane with an approximated speedometer velocity of $25 \mathrm{~km} / \mathrm{h}$. The SUV was located in the right lane with an approximated speedometer of $20 \mathrm{~km} / \mathrm{h}$. In the lower part of Fig. 6, we can see two photographs of the portable demonstrator setup, and in the upper part, a video frame of both vehicles approaching the radar is shown.

The range-time images obtained in the experiment are shown in Fig. 7. This range-time images correspond to the video frame presented in Fig. 6. We can clearly appreciate how actual vehicle scattering depart from the point target model used in the simulations. We can also see how stationary clutter (due to trees, traffic signs, road lighting ...) is present in the real scenario. However, we can appreciate how the phase fringes are still visible in the vehicles reflections shown in the range-time interferometric image. This is mainly explained because, for a high-resolution radar, a vehicle is composed by a set of pointtarget-like reflectors that will be likely resolved in range. This fact makes possible to extract a noncorrupted interferometric phase from the prominent scatter and make a correct road-lane detection using (12) as shown in Fig. 8. In Fig. 8, we can see how 


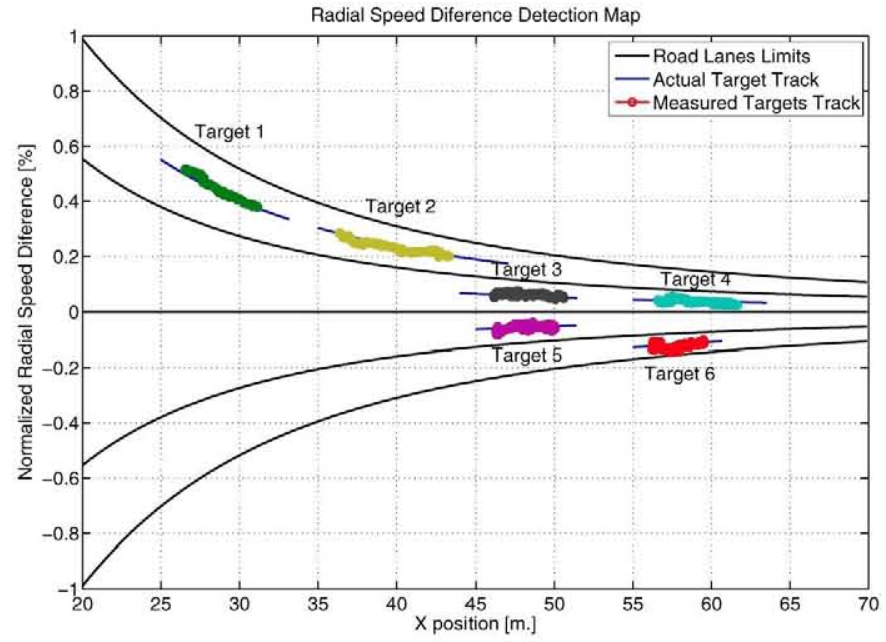

(a)

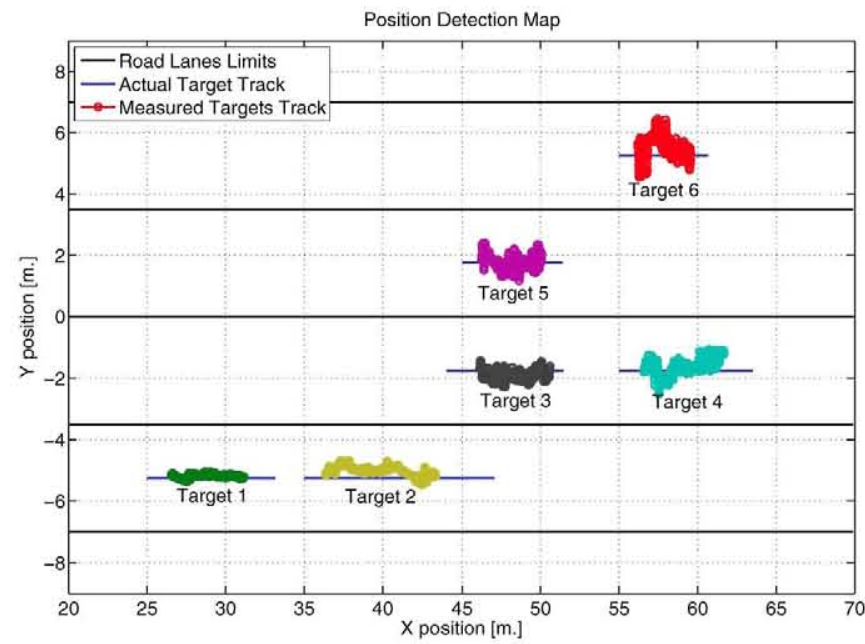

(b)

Fig. 3. DTR detection maps in a simulated scenario. Radial speed difference detection map is shown in (a). Position detection map is shown in (b).

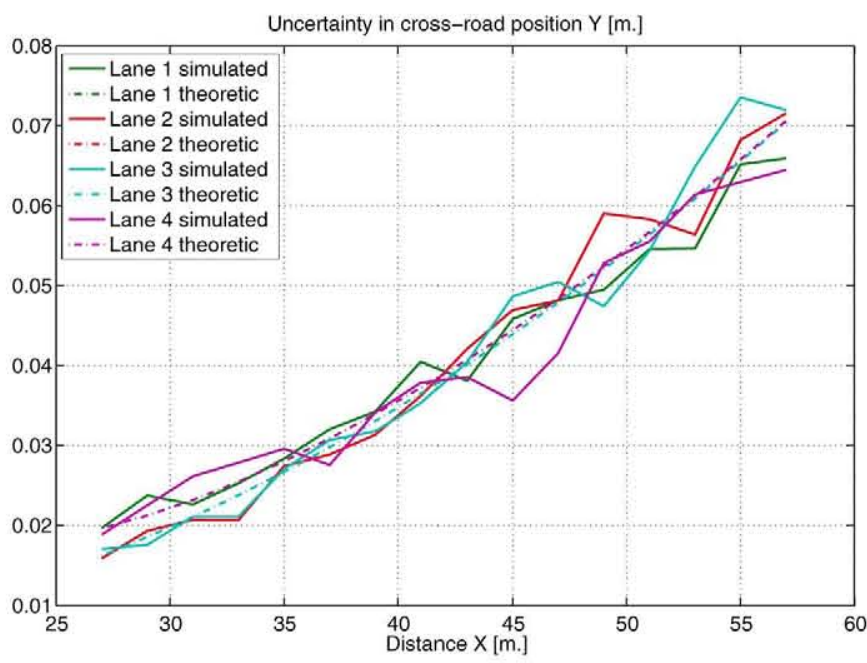

Fig. 4. DTR cross-road position uncertainty.

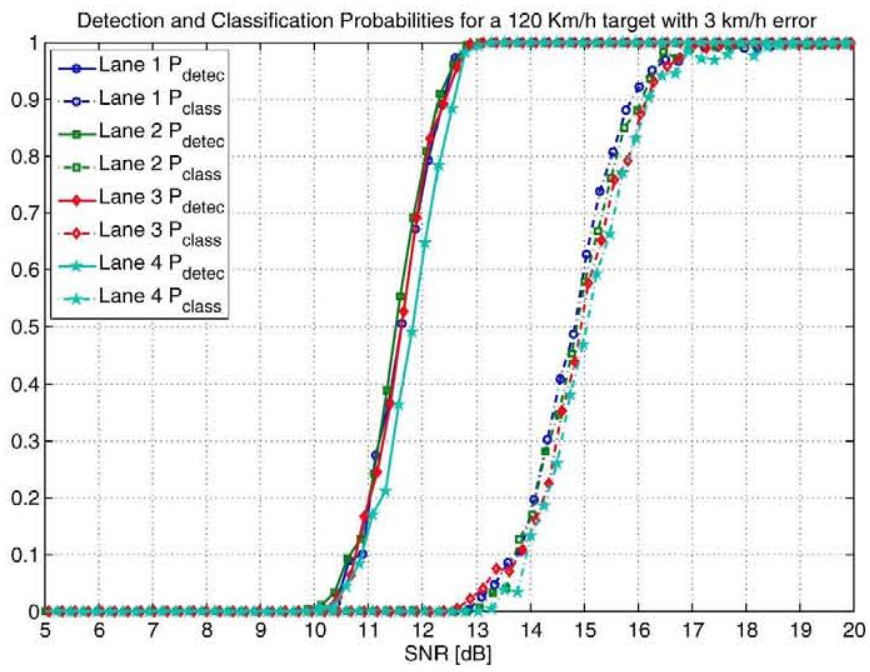

Fig. 5. DTR detection and classification probabilities against noise.

radial speed difference obtained with the interferometric phase extracted from the range-time images is translated into crossroad position. As in the simulations, radial speed difference

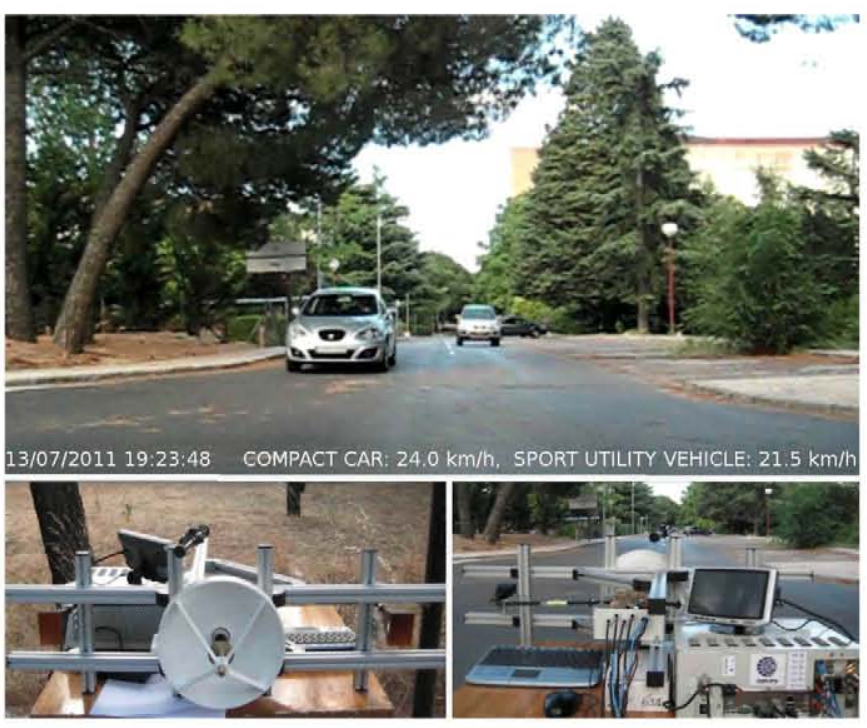

Fig. 6. DTR field trials setup and experiment video frame.

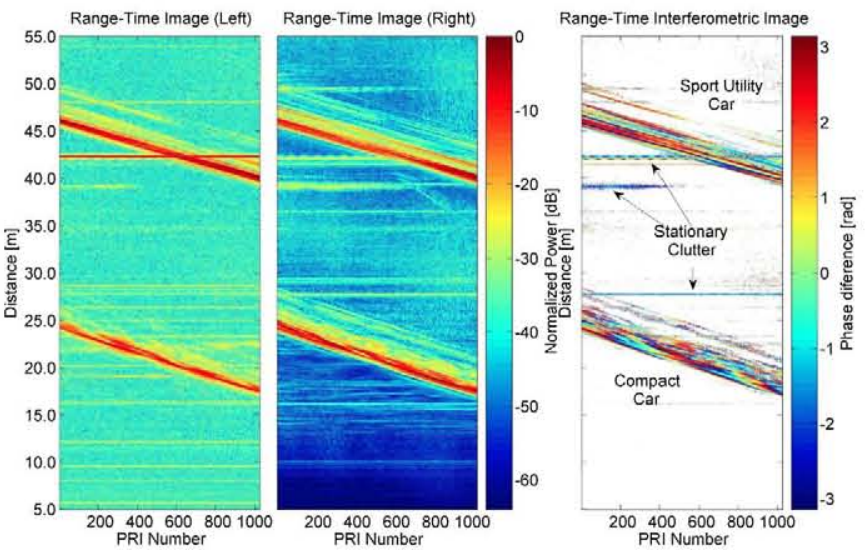

Fig. 7. DTR range-time images in a real experiment.

presents deviation from the theoretical one mainly due to the phase scintillation phenomenon which is not taken into account in the simulations results. However, the mean value of all the 


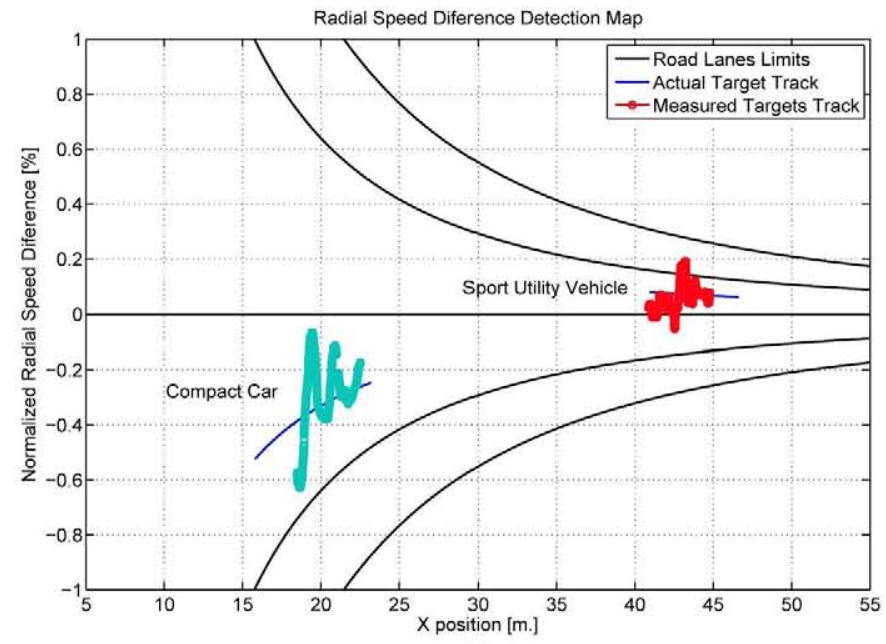

(a)

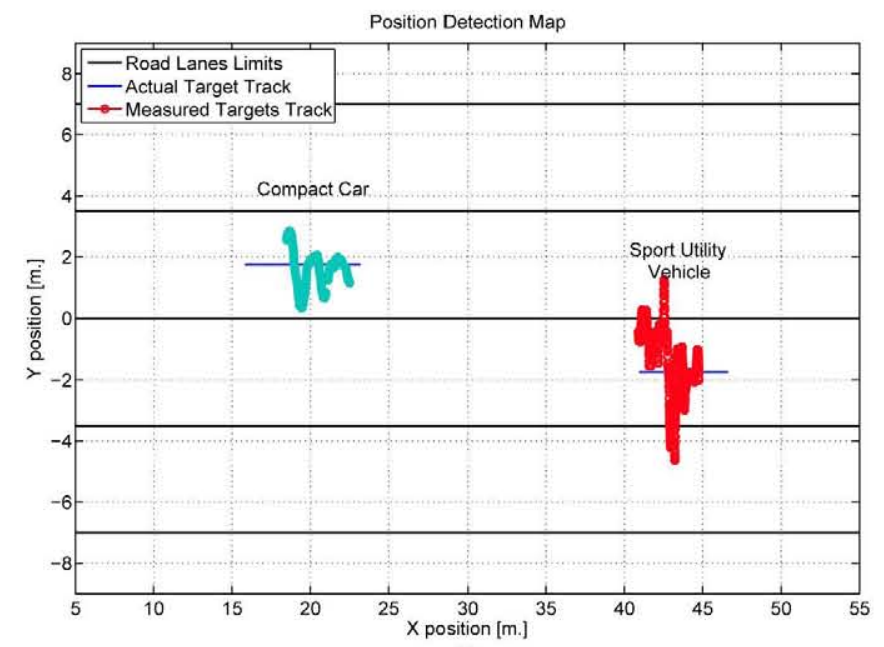

(b)

Fig. 8. DTR detection maps in a real experiment. Radial speed difference detection map is shown in (a). Position detection map is shown in (b).
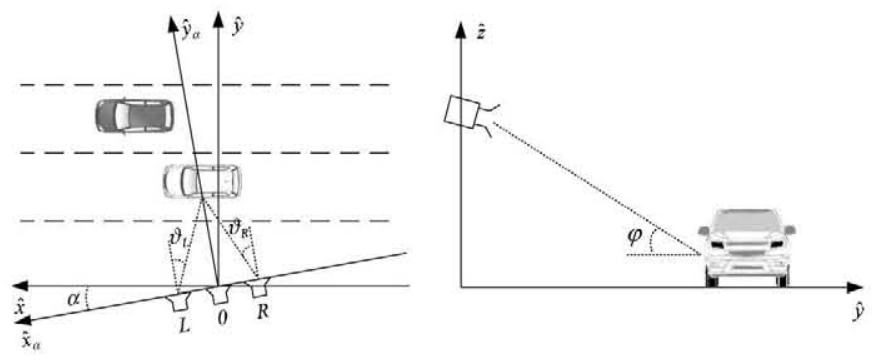

Fig. 9. Across-the-road detection geometry.

instantaneous measures, which is used to estimate the road lane, is still very close to the actual position. The detected speeds are shown in Fig. 6 and are slightly different to the theoretical ones. This fact could be explained due to the speedometer error or by uncontrolled deviation from the desired speed by the drivers. This same experiment were repeated several times, and the speed measures were very similar to the expected ones. As a conclusion, road lane position, range, and speed are correctly estimated, confirming the viability of this configuration in a true road scene.

\section{ACROSS-THE-ROAD CONFIGURATION}

\section{A. Geometry}

In ATR configuration, the axis of transmitting and receiving antennas is directed across the line of travel of the target vehicle. The direction of the road is represented with the $\hat{x}$ axis in Fig. 9. In our detection geometry, the transmitting antenna is located at $O$, and the receiving antennas are at $L$ and $R$. The azimuth angles of the target as viewed from the receiving antennas are $\theta_{L}$ and $\theta_{R}$, and the elevation angles are denoted by $\varphi_{L}$ and $\varphi_{R}$. The radar baseline can be slightly skewed from the road direction by an angle $\alpha$.

\section{B. Road Lane and Speed Detection}

In ATR configuration, the phase of the received signal in each antenna is used to detect the target vehicle speed. The distance from target to radar is used to detect the road lane. The absolute phase difference between the antennas, or interferometric phase is given by

$$
\phi_{I}(t)=\frac{2 \pi}{\lambda}\left(r_{L}(t)-r_{R}(t)\right) .
$$

Calculating the gradient of the interferometric phase, it can be seen that it is related to the difference in radial velocities between antennas

$$
\frac{\partial \phi_{I}(t)}{\partial t}=\frac{2 \pi}{\lambda}\left(\frac{\partial r_{L}(t)}{\partial t}-\frac{\partial r_{R}(t)}{\partial t}\right)=\frac{2 \pi}{\lambda}\left(v_{r_{L}}(t)-v_{r_{R}}(t)\right) \text {. }
$$

Radial velocity is ultimately related to the azimuth and elevation angles of the target (22), if a target with constant velocity and no acceleration is considered, which is a reasonable assumption for a small azimuth beam width

$v_{R}(t)=V_{x} \cos \alpha \sin \theta(t) \cos \varphi(t)+V_{x} \sin \alpha \cos \theta(t) \cos \varphi(t)$.

Considering the instant when the target is at the same distance from both receiving antennas, the elevation angles, as viewed from both antennas, will be equal, as shown in (23), below, and the azimuth angles will be identical in magnitude but with different sign, as in (24), shown below:

$$
\begin{aligned}
\varphi_{L}\left(t_{O}\right) & =\varphi_{R}\left(t_{O}\right)=\varphi\left(t_{O}\right) \\
\theta_{L}\left(t_{O}\right) & =-\theta_{R}\left(t_{O}\right) \\
\sin \theta_{L}(t) & =-\sin \theta_{R}(t) \simeq \frac{B \cos \alpha}{2 y} .
\end{aligned}
$$

Taking (22)-(24) into account, we get the difference in radial velocities in

$$
\begin{aligned}
v_{R_{L}}(t)-v_{R_{R}}(t)=V_{x} & \cos \alpha \sin \theta_{L}(t) \cos \varphi_{L}(t) \\
& -V_{x} \cos \alpha \sin \theta_{R}(t) \cos \varphi_{R}(t) .
\end{aligned}
$$

Hence, using (21), (25) and (26), an expression relating the phase difference gradient and the speed of the target vehicle can be obtained

$$
V_{x}=\frac{\lambda y}{2 \pi B \cos ^{2} \alpha \cos \varphi\left(t_{O}\right)} \frac{\partial \phi_{I}\left(t_{O}\right)}{\partial t} .
$$


In order to obtain the road lane, the cross-road position of the target is compared to the known cross-road position of the road lane limits. When $\alpha$ is small, the cross-road position can be obtained using (28), shown below, where $r$ is the distance measured by the radar in any of the antennas and $h$ is the height of the radar

$$
y \simeq \sqrt{r^{2}-h^{2}} \cos \alpha .
$$

\section{Uncertainty in Road Lane Measurement}

1) Quantitative Analysis: The uncertainty of speed estimation will be affected by the uncertainty in phase difference, $x$ position, elevation angle, and central wavelength. Assuming the uncertainty sources are linearly independent, the total uncertainty is given by

$$
\begin{aligned}
\sigma_{V_{x}}^{2}=\left|\frac{\partial V_{x}}{\partial \dot{\phi}_{I}}\right|^{2} \sigma_{\dot{\phi}_{I}}^{2}+\left|\frac{\partial V_{x}}{\partial y}\right|^{2} & \sigma_{y}^{2}+\left|\frac{\partial V_{x}}{\partial \varphi}\right|^{2} \sigma_{\varphi}^{2} \\
& +\left|\frac{\partial V_{x}}{\partial \alpha}\right|^{2} \sigma_{\alpha}^{2}+\left|\frac{\partial V_{x}}{\partial \lambda}\right|^{2} \sigma_{\lambda}^{2} .
\end{aligned}
$$

Using (27), we finally obtain

$$
\begin{aligned}
\sigma_{V_{x}}^{2}= & \left|\frac{\lambda y}{2 \pi B \cos ^{2} \alpha \cos \varphi}\right|^{2} \sigma_{\dot{\phi}_{I}}^{2}+\left|\frac{V_{x}}{y}\right|^{2} \sigma_{y}^{2} \\
& +\left|V_{x} \tan \varphi\right|^{2} \sigma_{\varphi}^{2}+\left|2 V_{x} \tan \alpha\right|^{2} \sigma_{\alpha}^{2}+\left|\frac{V_{x}}{\lambda}\right|^{2} \sigma_{\lambda}^{2} .
\end{aligned}
$$

In addition to these uncertainties, there is another one related to the calibration process used to periodically remove any constant error [17].

2) Second-Order Effects: The uncertainty in the speed measurement is computed using (30). As in the DTR section, we will focus our analysis in the term related to the uncertainty in the phase difference measurement, which is the only term that is unique to interferometric radars. Due to the approximations made in formulas derivation, some errors affecting sensitivity are still present and cannot be evaluated in a quantitative way. However, these errors can still be analyzed in a qualitative way. The main geometrical assumption is that the target is supposed to be exactly in front of the radar beam, which is true in a certain instant but false in all the time-on-target. However, this is not a limitation if a narrow beam is used, which can be perfectly achieved in the millimeter wave band. As in the DTR section, in previous formulas, a target with constant velocity, and no acceleration has been considered, which is typical for the small dwell time obtained with the small beam widths of millimeter wave radars. When the zero acceleration assumption is not met, it will produce a slight variation in radial velocity difference and, as a result, an increase in system uncertainties.

\section{Operational Limits}

In order to obtain the gradient of interferometric phase, that we need to estimate the speed with (27), the phase change between consecutive samples must be smaller than $\pi$ in order to avoid phase aliasing. This condition will limit the maximum speed of a target to correctly measure it. Using (27), and replacing the continuous phase derivative by time and phase increments, we obtain

$$
\begin{aligned}
V_{x} & =\frac{\lambda y}{2 \pi B \cos ^{2} \alpha \cos \varphi\left(t_{O}\right)} \frac{\Delta \phi_{I}}{\Delta t} \\
\Delta t & =\frac{1}{R R F} .
\end{aligned}
$$

The restriction to correctly estimate the phase gradient is having two samples in each phase cycle. However, in order to achieve the best performance of this technique, and reduce phase difference variance, it is better to have more samples per phase cycle

$$
\Delta \phi_{I}=\frac{2 \pi}{N_{S}} .
$$

If we consider $N_{S}$ samples per phase cycle, the maximum speed to correctly estimate the road lane will be given by

$$
V_{x \max } \simeq \frac{\lambda y R R F}{B \cos ^{2} \alpha \cos \varphi\left(t_{O}\right) N_{S}} .
$$

Another limitation of this configuration is the time the target vehicle is in the radar beam. This time must be long enough to collect $N_{S}$ phase samples as before. This number of samples is related to the target speed and the radar beam width $\theta_{a z}$

$$
N_{S}=\frac{2 \tan \left(\frac{\theta_{a z}}{2}\right) y}{V_{x}} R R F \simeq \frac{\theta_{a z} y}{V_{x}} R R F .
$$

Finally, considering $N_{S}$ samples per phase cycle, the maximum speed to correctly estimate the road lane is the minimum of the speeds given by (34) and

$$
V_{x \max }=\frac{y \theta_{a z}}{N_{S}} R R F
$$

Some conclusions can be stated when looking at (34). A higher detectable velocity is possible for remote lanes compared to close ones. Increasing the ramp repetition frequency or the wavelength, or decreasing the baseline or the number of samples per phase cycle, will increase the maximum detectable velocity. However, they will have a different effect in the uncertainties, and therefore, a tradeoff must be reached.

\section{E. Simulation Results}

IN this section, three kind of simulation results are presented. In first place, we will show the correct performance of the proposed interferometric ATR configuration in a dense traffic scenario. This simulation is also used to illustrate the signal processing chain. In second place, we will validate the analytic formula for the uncertainty in the along-the-road velocity measurement with a Monte Carlo simulation. Finally, we will use another Monte Carlo simulation to show the performance against noise. The simulation scenario is the same as in the DTR configuration, but now the radar is placed outside instead of being above the road. The baseline is aligned with the road direction for this simulation; therefore, $\alpha=0^{\circ}$. The actual position and speeds of the targets can be seen in Table II, along with the results of the detected position, speed, and road lane.

The first stage of the signal processing chain is to obtain a range-time image of the scene from each of the receiving 
TABLE II

ATR Simulated SCEnARIO CHARACTERISTICSAND RESUlts

\begin{tabular}{ccccccc}
\hline \hline $\begin{array}{c}\text { Target } \\
\text { number }\end{array}$ & $\begin{array}{c}\text { Actual } \\
\text { Speed } \\
(\mathrm{Km} / \mathrm{h})\end{array}$ & $\begin{array}{c}\text { Actual } \\
\text { cross-road } \\
\text { position }(\mathrm{m})\end{array}$ & $\begin{array}{c}\text { Actual } \\
\text { Lane }\end{array}$ & $\begin{array}{c}\text { Detected } \\
\text { Speed } \\
(\mathrm{Km} / \mathrm{h})\end{array}$ & $\begin{array}{c}\text { Detected } \\
\text { cross-road } \\
\text { position }(\mathrm{m})\end{array}$ & $\begin{array}{c}\text { Detected } \\
\text { Lane }\end{array}$ \\
\hline 1 & 115.0 & +15.25 & 4 & 115.40 & +15.26 & 4 \\
2 & 170.0 & +15.25 & 4 & 167.28 & +15.26 & 4 \\
3 & 105.0 & +11.75 & 3 & 105.21 & +11.76 & 3 \\
4 & 120.0 & +11.75 & 3 & 119.77 & +11.76 & 3 \\
5 & 90.0 & +8.25 & 2 & 89.91 & +8.26 & 2 \\
6 & 80.0 & +4.75 & 1 & 79.93 & +4.76 & 1 \\
\hline
\end{tabular}

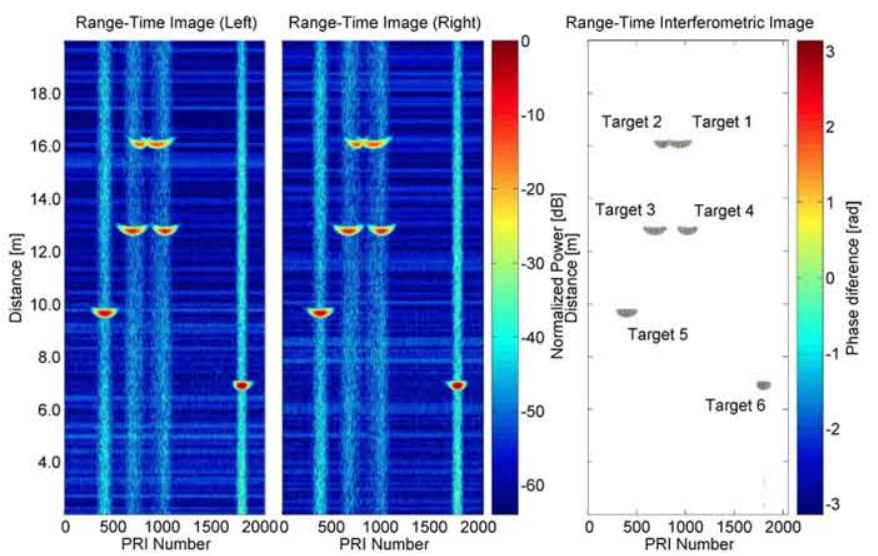

Fig. 10. ATR range-time images in a simulated scenario.

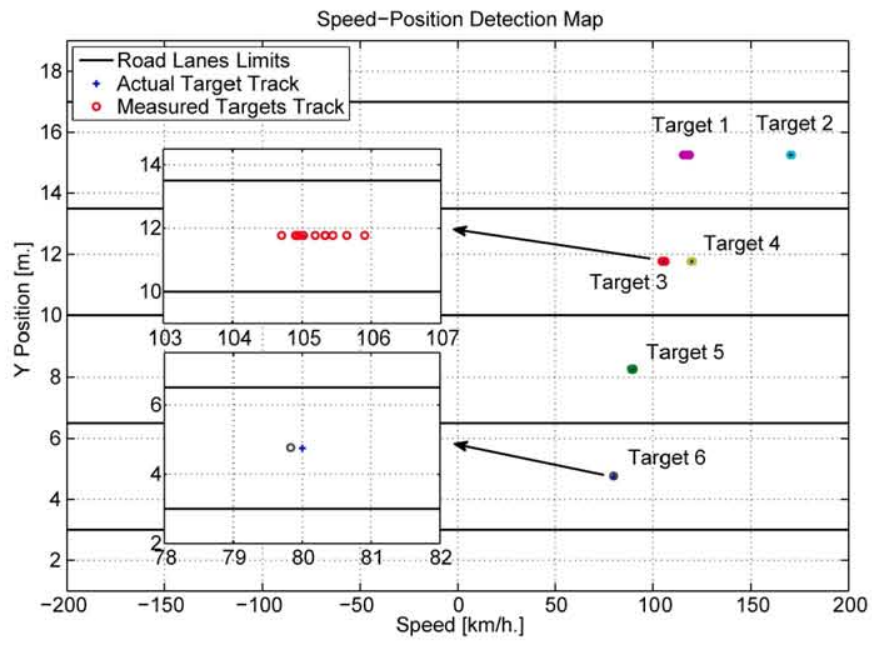

Fig. 11. ATR simulated scenario and detected targets.

antennas. The images for our example are shown in Fig. 10. These images will allow us to estimate the road lane and obtain the phase difference in order to estimate the along-the-road speed. In ATR configuration, it is not necessary to isolate the targets because they are already isolated with a narrow antenna beam width pointed in cross-road direction. Just one target can be at a certain distance inside the radar beam. However, many targets may be inside the radar beam if they travel in different road lanes, but they can be discriminated in distance. Road lane estimation is a trivial issue, using (28), just knowing the targets distance. Once the interferometric phase is extracted, the estimation of the along-the-road velocity is also straight forward using (27).

A detail of the speed estimation and the road lane detection map are shown in Fig. 11. The limits of the road lanes are

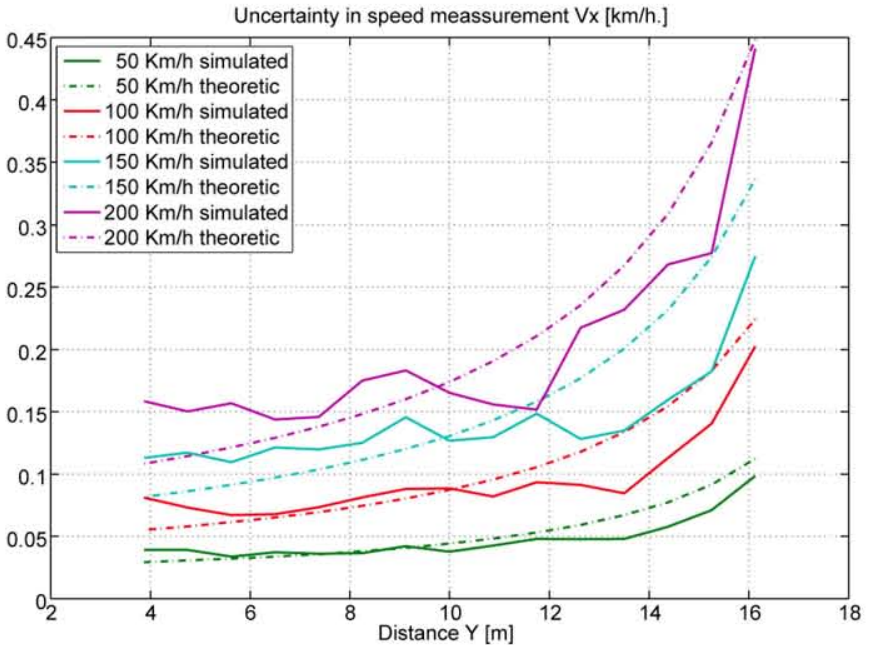

Fig. 12. ATR speed-measurement uncertainty,

plotted in black, the actual target value is plotted in blue, and the detected target value is plotted with a different color for each target. In this figure, all speed values detected in the dwell time are plotted to give an idea of the measure dispersion. However, the speed value is estimated using the mean of all those measurements. As in DTR, Fig. 11 can show many of the reasonings made in Section III-C. Specifically, targets at longer distances have more dispersion in speed measurement than the closer ones. In order to validate the analytic formula for the uncertainty in the along-the-road velocity measurement, a Monte Carlo simulation has been run. In this simulation, we focus on the dependence of speed uncertainty with the distance to the radar, which is the predominant term of the factor associated to the phase difference gradient uncertainty. The simulation has been repeated for four different velocities. In Fig. 12, it can be seen how the analytic formula fits very well with the obtained simulation results. The baseline used in the simulations is $B=0.77 \mathrm{~m}$. The central wavelength is $\lambda=8.6 e-$ $3 \mathrm{~m}$, and the local oscillator has a typical phase error deviation of $\sigma_{\Phi}=0.1897 \mathrm{rad}$, which leads to a deviation in the interferometric phase difference of $\sigma_{\dot{\phi}_{I}}=0.3794 \mathrm{rad} / \mathrm{s}$. The standard deviation in distance measurement is $\sigma_{y}=1.8 e^{-2} \mathrm{~m}$. The deviation of the central wavelength, deviation of the elevation angle, and deviation of the skew angle are neglected in the simulations. The performance of the ATR technique against noise is shown in Fig. 13. Two kinds of curves are obtained using simulation, probability of detection and classification against SNR. The SNR is defined in the time domain as the relation of the peak returned echo power and the noise power. In the ATR configuration, interferometry is used to estimate the speed. We considered that a target is detected when we know about its presence and the road lane is correctly detected. A target is classified when it is detected, the road lane is correctly estimated, and the speed error is below a threshold. In Fig. 13(a), we can clearly appreciate that the probability of detection is higher for the slower targets because the time-ontarget, and consequently the received energy from that target, is higher. The energy above the detection threshold that we need to correctly classify a target depends on the maximum error allowed for the speed measurement, this is clearly illustrated 


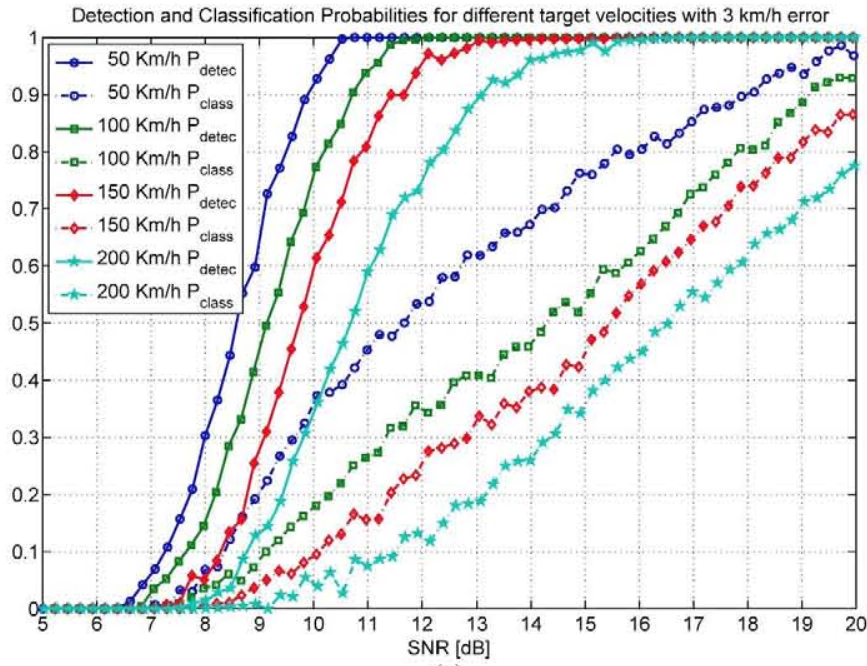

(a)

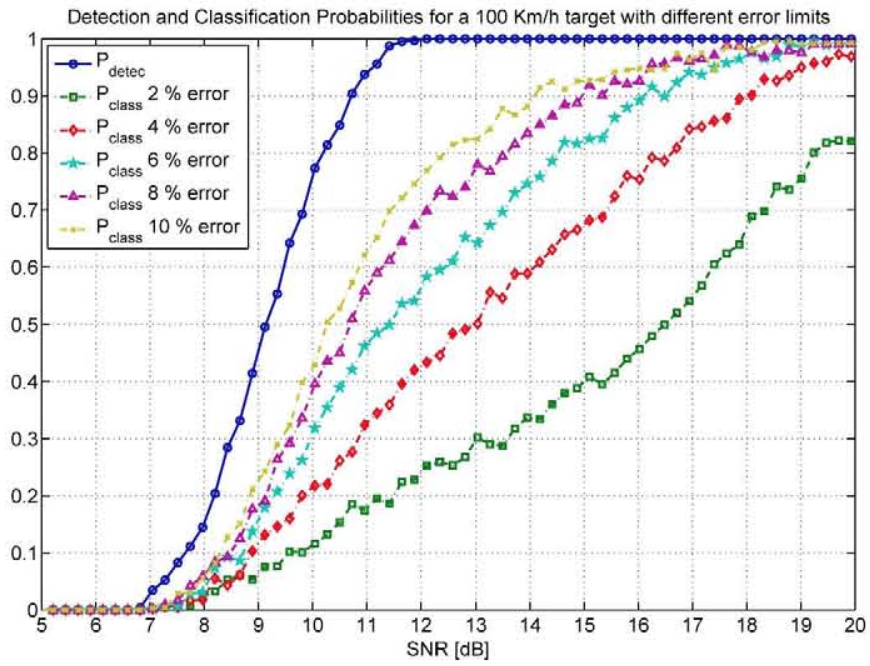

(b)

Fig. 13. ATR detection and classification probabilities against noise. The effect on performance of the target speed is shown in (a). The effect on performance of the speed error limit is shown in (b).

in Fig. 13(b). For a $100-\mathrm{Km} / \mathrm{h}$ target and a maximum error of $10 \mathrm{Km} / \mathrm{h}$, we need a $2-\mathrm{dB}$ higher SNR, but if we prefer a $2-\mathrm{Km} / \mathrm{h}$ error, we need a $10-\mathrm{dB}$ increase in SNR.

\section{F. Experimental Results}

The characteristics of our radar demonstrator fit better with the ATR configuration due to the narrow azimuth beam widths and suitable baseline. The experiments for the ATR configuration were conducted in a controlled traffic scene. Transmitted bandwidth, baseline, and beam width are the same as in the DTR configuration. Ramp repetition frequency has been increased to $2000 \mathrm{~Hz}$ to have more samples from the target in each phase cycle as shown in (35). The field trial were conducted in a controlled traffic parking shown in the upper part of Fig. 14. The car used for the field trials was the SUV. The SUV was driving from the left to the right of the scene at a speed of approximately $20 \mathrm{~km} / \mathrm{h}$. In the lower part of Fig. 14, we can see two photographs of the portable demonstrator setup. The baseline of the radar was skewed with an angle of $\alpha=-10^{\circ}$ from the vehicle velocity direction. This

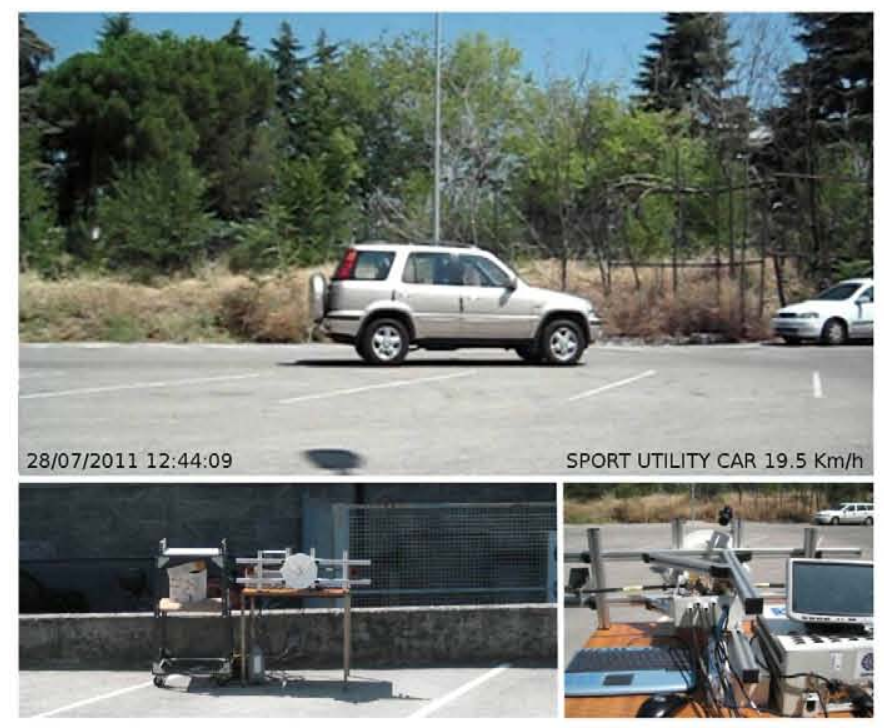

Fig. 14. ATR field trials setup and experiment video frame.

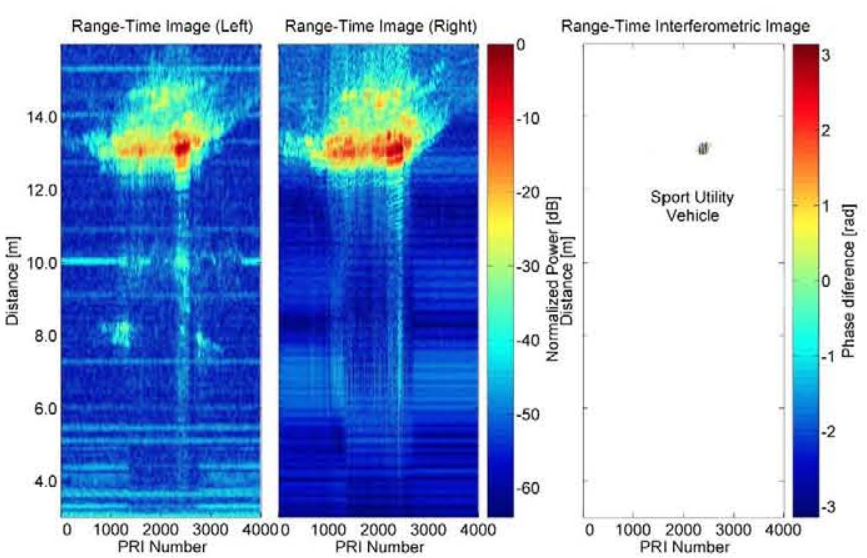

Fig. 15. ATR range-time images in a real experiment.

is necessary to avoid the specular reflections that occasionally saturated the receiver corrupting the interferometric phase. This angle is taken into account to measure the true along-the-road speed of the target, as we saw in (27). The range-time images obtained in the experiment are shown in Fig. 15. As in the DTR experiment, the range-time images correspond to the presented video frame. As in DTR, actual vehicle scattering departs from that shown in simulations for point targets. However, as we see in the images in Fig. 15, the behavior of the strongest return presents a nice phase fringe pattern in the interferometric image. Interferometric phase can be extracted from that image, as done in the simulations, and the speed can be estimated using (27). The results of the detected speed and lane position are shown in Fig. 16.

As in the simulation result section, in Fig. 16, all instantaneous speed measures values are plotted to give an idea of the measure dispersion. However, the speed value is estimated using the mean value of all those instantaneous values.

Similar uncertainty in the experimental results is observed when they are compared to the simulation results. Hence, road lane position and speed were correctly estimated confirming the viability of this configuration. 


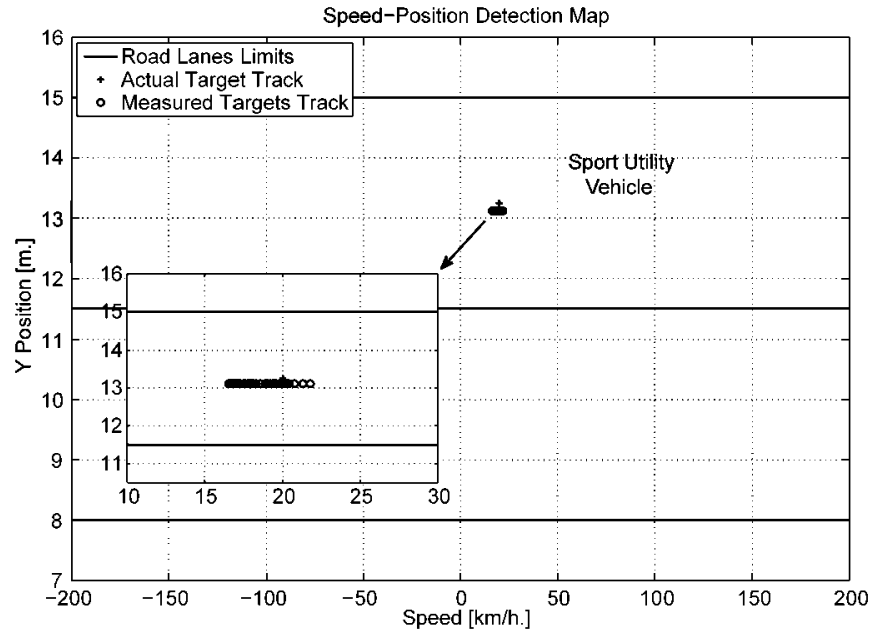

Fig. 16. ATR real experiment scenario and detected targets.

\section{CONCLUSION}

In this paper, we have presented two different interferometric systems that can simultaneously measure speed, range, and road lane position of several vehicles. In DTR configuration, interferometry is used to estimate road lane position. Without the use of interferometry, we are not able to identify the road lane of the speeding car in case more than one were inside the radar beam. In ATR configuration, interferometry is used to estimate target speed that otherwise could not be done when the radar beam is pointing nearly perpendicular to the traffic direction. The feasibility of the proposed techniques has been successfully demonstrated with simulations and field trials in a controlled road scene using a Ka-band interferometric radar.

However, the characteristics of our demonstrator are far for been optimum for both configurations in a real traffic environment. Hence, as a conclusion to this study, we are going to indicate some characteristics and relations that the optimum system should met for each configuration. In DTR configuration, the radar would be placed above the road mounted in a traffic sign or in crossing bridge. Typical height could be 4 to $5 \mathrm{~m}$. Taking this into account, an elevation angle whose cosine is very near to one should be chosen in order to satisfy the conditions for the approximations done in (6). However, it is interesting to reduce as much as possible the detection range to enhance the radial speed difference between the antennas and, of course, the SNR. An optimal central distance could be $45 \mathrm{~m}$, as used in the simulations, which gives a cosine error of less than $0.6 \%$. An important point is that the radar must illuminate all highway lanes. If a four lane highway is considered, the system must cover at least 10 to $12 \mathrm{~m}$ in cross-road direction and at around $20 \mathrm{~m}$ in DTR; therefore, a $15-20^{\circ}$ beam width will perfectly fit. In order to optimize system performance, a high baseline should be selected to reduce the uncertainty, and also a high ramp repetition frequency to isolate the targets easily. A relation between baseline and RRF could be obtained from (19), using the desired maximum detectable speed.

In ATR configuration, the radar would be placed outside the road mounted in a separate mast. Its position must be high enough to guarantee good visibility without concealing the road lanes, even when several targets are simultaneously in front of the radar position in different lanes. Typical height could be 5 to $6 \mathrm{~m}$. Taking this into account, an elevation beam width that illuminate all the lanes of the highway from the location of the radar must be chosen. If a four lane highway is considered, the system must cover at least 10 to $12 \mathrm{~m}$ in crossroad direction; therefore, a $25-30^{\circ}$ beam width will provide a uniform illumination for all road lanes. However, in the azimuth direction is better to have quite narrow beam widths in order to avoid having multiple targets at the same distance into the radar beam. A $6^{\circ}$ beam width should be enough for short distances. In order to optimize system performance, a high baseline should be selected to reduce uncertainty, as well as a high ramp repetition frequency to increase the number of speed measures with a constant dwell time. A relation between baseline and RRF could be obtained from (34) and (36) using the desired maximum detectable speed.

\section{REFERENCES}

[1] G. Wang, D. Xiao, and J. Gu, "Review on vehicle detection based on video for traffic surveillance," in Proc. IEEE ICAL, Sep. 2008, pp. 2961-2966.

[2] M. Papageorgiou, C. Diakaki, V. Dinopoulou, A. Kotsialos, and Y. Wang, "Review of road traffic control strategies," Proc. IEEE, vol. 91, no. 12, pp. 2043-2067, Dec. 2003.

[3] V. Bhatt, S. Khati, D. Pandey, and H. Pant, "Wireless traffic system with speed control," in Proc. 2nd ICCAE, Feb. 2010, vol. 1, pp. 413-417.

[4] S. Suchandt, H. Runge, H. Breit, U. Steinbrecher, A. Kotenkov, and U. Balss, "Automatic extraction of traffic flows using terrasar-x alongtrack interferometry," IEEE Trans. Geosci. Remote Sens., vol. 48, no. 2, pp. 807-819, Feb. 2010.

[5] J. Jendzurski and N. G. Paulter, "Calibration of speed enforcement downthe-road radars," J. Res. Nat. Inst. Std. Technol., vol. 114, no. 3, pp. 137148, May/Jun. 2009.

[6] R. Westphal and A. Kessler, “35-ghz-doppler radar for law enforcement agencies in europe," in IEEE MTT-S Int. Microw. Symp. Dig., May 1988, vol. 2, pp. 1031-1033.

[7] P. Fisher, "Improving on police radar," IEEE Spectr, vol. 29, no. 7, pp. 38 43, Jul. 1992.

[8] T. K. Ishii, "Analysis of target-speed determination with doppler radar," IEEE Trans. Instrum. Meas., vol. IM-19, no. 2, pp. 86-91, May 1970.

[9] C. Weil, D. Camell, D. Novotny, and R. Johnk, "Across-the-road photo traffic radars: New calibration techniques," in Proc. 15th Int. Conf. Microw., Radar Wireless Communications, MIKON, May 2004, vol. 3 , pp. 889-892.

[10] P. Fisher and J. Pyhtila, "Application of laser speed-measurement technologies in highway-safety programs," in Proc. 29th Symp. Autom. Technol. Autom., Jun. 1996, vol. 3, pp. 297-304.

[11] P. Fisher and J. Pyhtila, "Timing quantization error in lidar speedmeasurement devices," IEEE Trans. Veh. Technol., vol. 49, no. 1, pp. 276280 , Jan. 2000.

[12] J. Munoz-Ferreras, J. Calvo-Gallego, and F. Perez-Martinez, "Monitoring road traffic with a high resolution lfmcw radar," in Proc. IEEE RADAR Conf., May 2008, pp. 1-5.

[13] J. Munoz-Ferreras, F. Perez-Martinez, J. Calvo-Gallego, A. Asensio-Lopez, B. Dorta-Naranjo, and A. B. del Campo, "Traffic surveillance system based on a high-resolution radar," IEEE Trans. Geosci. Remote Sens., vol. 46, no. 6, pp. 1624-1633, Jun. 2008.

[14] J. Woll, "Monopulse doppler radar for vehicle applications," in Proc. Intell. Veh. Symp., Sep. 1995, pp. 42-47.

[15] D. Felguera-Martín, J.-T. González-Partida, P. Almorox-González, M. Burgos-García, and B.-P. Dorta-Naranjo, "Interferometric inverse synthetic aperture radar experiment using an interferometric linear frequency modulated continuous wave millimetre-wave radar," IET Radar, Sonar Navig., vol. 5, no. 1, pp. 39-47, Jan. 2011.

[16] J. Wang and D. Kasilingam, "Global range alignment for ISAR," IEEE Trans. Aerosp. Electron. Syst., vol. 39, no. 1, pp. 351-357, Jan. 2003.

[17] C. M. Weil, Calibration techniques for across-the-road traffic radars, Nat. Inst. Std. Technol., May 1998. NIST Tech. Note 1398. 


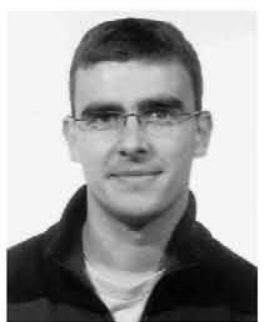

David Felguera-Martín was born in Madrid, Spain, in 1982. He received the Ingeniero de Telecomunicación degree from the Universidad Politécnica de Madrid in 2005, where he is currently working toward the Ph.D. degree.

His research activities are in the area of multimode radar systems and radar signal processing, particularly Maritime and Ground Moving Target Indicators, synthetic aperture radar, and radar interferometry.

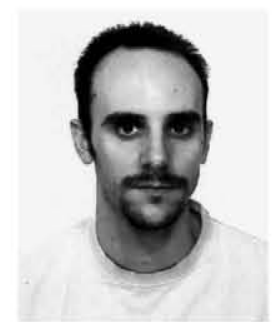

José-Tomás González-Partida was born in Madrid, Spain, in 1981. He received the Telecommunication Engineering and the Ph.D. degrees from the Universidad Politécnica de Madrid (UPM) in 2004 and 2009 , respectively.

Since September 2003, he has been with the Microwave and Radar Group, Department of Signals, Systems, and Radiocommunications, UPM, where he is currently an Assistant Professor. His research activities are in the area of radar systems, specifically high-resolution applications, millimeter-wave radars, synthetic aperture radar, and radar interferometry.

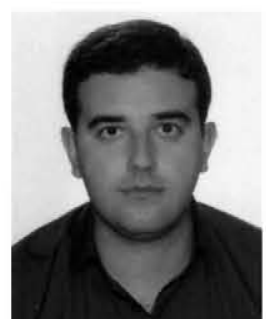

Pablo Almorox-González was born in Ponferrada (León), Spain, in 1980. He received the Ingeniero de Telecomunicación degree from the Universidad Politécnica de Madrid (UPM), Madrid, Spain, in 2004, where he is currently working toward the Ph.D. degree.

Since September 2003, he has been with the Grupo de Microondas y Radar, Departamento de Señales, Sistemas y Radiocomunicaciones, UPM. His research activities are in the areas of radar systems, specifically the design and characterization of highresolution radars, and microwave and millimeter-wave subsystems.

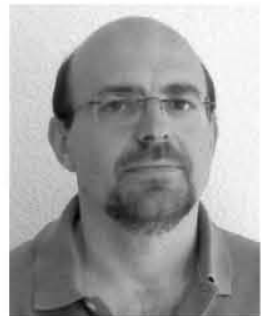

Mateo Burgos-García received the degree in telecommunications engineering in 1988 and the $\mathrm{Ph} . \mathrm{D}$. degree in 1994, both from the Polytechnical University of Madrid (UPM), Madrid, Spain.

He has been an Assistant Professor with UPM since 1991 and Full Professor since 2010. His research activities include high-resolution radars and the associated signal processing for radar and spectrum surveillance applications. His second line of research is advanced applications in the millimeterwave and Teraherz bands. This includes the development of high-resolution smart radar sensors, passive imaging, and through-the-wall surveillance.

Dr. Burgos-García is member of the Microwaves and Radar Group of the Telecommunications Engineering Technical School, UPM. 